\title{
Mereology and Identity
}

\section{Massimiliano Carrara ${ }^{1} \cdot{\text { Giorgio } \text { Lando }^{2} \text { (D }}$}

Received: 14 February 2020 / Accepted: 18 February 2020 / Published online: 2 March 2020

(c) Springer Nature B.V. 2020

\section{Introduction}

The eighteen papers in this special issue scrutinise the multifarious roles that identity plays in mereology. In this introduction, we present an overview of the topics at stake.

Specifically, in Sect. 2 we characterize in general the main points of contact between mereology and the philosophical problems raised by identity. In Sect. 3 we deal with the problem of whether mereology enjoys a status of topic neutrality and overall logicality analogous to that usually attributed to identity. We also discuss the connected epistemological problem of the alleged analyticity of some mereological principles. This section presents the papers by Aaron Cotnoir, Jean-Baptiste Guillon, Paolo Maffezioli \& Achille Varzi, Lorenzo Azzano and Meg Wallace. In Sect. 4 we focus on mereological extensionality, discussing in particular the paper by Jeroen Smid. In Sect. 5 we ask whether mereological principles can explain or ground other principles, or are in turn in need of an explanation or ground, and we show that similar problems can arise about identity. The papers by Joshua Spencer and Phillip Bricker are presented in this section. In Sect. 6 we compare the modal status of identity with the seemingly different modal status of mereological relations, and we present in particular Manuel Lechthaler's paper and our own paper. In the last two Sects. 7, 8, we cover the papers that more directly concern the so-called thesis of Composition as Identity. In Sect. 7 we show that some papers raise new issues for Composition as Identity and other related philosophical theses. The contributions by Martin Lipman, Byeong-uk Yi and Claudio Calosi belong to this group. Finally, in Sect. 8, we introduce the new varieties of Composition as Identity and some new reflections on its methodological aspects,

Giorgio Lando

giorgio.lando@univaq.it

Massimiliano Carrara

massimiliano.carrara@unipd.it

1 Dipartimento di Filosofia, Sociologia, Pedagogia e Psicologia Applicata - Sezione di Filosofia, Università degli Studi di Padova, Piazza Capitaniato 3, 35139 Padova, Italy

2 Dipartimento di Scienze Umane, Università degli Studi dell'Aquila, Viale Nizza 14, 67100 L'Aquila, Italy 
with a special focus on the papers by Roberto Loss, Martina Botti, Jonathan Payton, Einar Bøhn and Evan Woods.

\section{The roles of identity in mereology}

Mereology is the discipline that investigates the features of the relation of parthood $(P)$ and of some other connected relations, such as proper parthood $(P P)$, overlap (o) and composition $(\Sigma)$. Parthood is taken as a primitive relation. According to the standard definitions of the other relations in terms of it, $x$ is a proper part of $y$ if and only if $x$ is a part of $y$ and $x$ is not identical to $y$; $x$ overlaps $y$ if and only if they have at least a part in common; some $x x$ (where $x x, y y, z z, \ldots$ are plural variables) compose a whole $y$ if and only if each of $x x$ is part of $y$ and every part of $y$ overlaps at least one of $(\prec) x x$ :

$$
\begin{array}{lr}
x P P y \equiv_{\operatorname{def}} x P y \wedge x \neq y & \text { (Proper Parthood - Definition) } \\
x \circ y \equiv_{\text {def }} \exists z(z P x \wedge z P y) & \text { (Overlap - Definition) } \\
x x \Sigma y \equiv_{\text {def }} \forall z(z \prec x x \rightarrow z P y) \wedge \forall z(z P y \rightarrow & \exists w(w \circ z \wedge w \prec x x)) \\
& \text { (Composition - Definition) }
\end{array}
$$

The features of these relations can be characterised by a theory, that is, by a set of theorems, which follow from some axioms. A specific theory, namely Classical Extensional Mereology (CEM), was dominant in the twentieth century, and several important philosophers such as Goodman, Quine and Lewis believed that CEM characterizes parthood and the other cognate relations in a sound and exhaustive way. CEM can be derived from three simple axioms, namely Transitivity of Parthood, Uniqueness of Composition and Unrestricted Composition:

$$
\begin{aligned}
& \forall x \forall y \forall z(x P y \wedge y P z \rightarrow x P z) \\
& \forall x x \forall y(x x \Sigma y \rightarrow \forall z(x x \Sigma z \rightarrow z=y)) \\
& \forall x x \exists y(x x \Sigma y)
\end{aligned}
$$

(Transitivity of Parthood) (Uniqueness of Composition) (Unrestricted Composition)

Uniqueness of Composition and Unrestricted Composition jointly establish that for every plurality of entities there is exactly one entity composed by these entities. CEM includes as theorems several other principles of utmost philosophical interest, such as Weak Supplementation, Strong Supplementation and Extensionality:

$$
\begin{array}{lr}
\forall x \forall y(x P P y \rightarrow \exists z(\neg z \circ x \wedge z P y)) & \text { (Weak Supplementation) } \\
\forall x \forall y(\neg x P y \rightarrow \exists z(z P x \wedge \neg z \circ y)) & \text { (Strong Supplementation) } \\
\forall x \forall y((\exists z(z P P) \wedge \exists z(z P P y)) \rightarrow(\forall z(z P P x \leftrightarrow z P P y) \leftrightarrow x=y)) & \\
\text { (Extensionality) } &
\end{array}
$$

Weak Supplementation dictates_roughly - that nothing has a single proper part. Strong Supllementation establishes that, if $x$ is not a part of $y$, then there is something in $x$ which does not overlap $y$. Extensionality sets forth identity conditions for every entity having proper parts: they are identical if and only if they have the same proper parts.

These controversial principles of CEM are mutually connected in a variety of ways. Several alternative theories of parthood-non-classical mereologies—are currently 
being explored. They are in most cases weaker than CEM, and drop some of its controversial principles, often disentangling them one from another.

Extensionality is perhaps the most ambitious and controversial among the controversial principles of CEM, inasmuch as it purports to provide identity conditions for the large majority of entities in general (only entities without proper parts-i.e., atomic entities - are out of its scope of application). Extensionality follows from the axiom of the Uniqueness of Composition, and forbids any distinction of entities without an underlying difference in what is in them (i.e., their proper parts). As a consequence, Extensionality is a powerful engine of ontological economy and was in consonance with the nominalistic inclinations of the above mentioned original backers of CEM.

Non-classical mereologies are sometimes propelled by metaphysical stances (such as dualism about constitution), which are incompatible with these austere forms of ontological economy. Some non-classical mereologies are driven by the idea that parthood and cognate relations should not be expected to work in the same way for every category of entities: general metaphysical divides, such as the abstract/concrete distinction and the artifactual/natural entities distinction, would also have an impact on the features of the mereological relations (see Moltmann (1997) for a typical approach of this kind). According to some scholars, even less general distinctions require a plurality of mereologies: for example, in the extreme case of (Llored and Harré 2014), even the mereology of chemistry should be pluralistic (i.e., the various entities that chemistry is about would require different mereologies).

Extensionality is about identity: it is the way in which CEM provides identity conditions for every complex entity. Identity is also connected with composition. Owing to Extensionality and to the ensuing rejection of distinctions of entities without a distinction in their proper parts, it seems that (in a sense to be clarified) any composed entity is nothing more than its components. This led some philosophers, such as Lewis, Armstrong and Baxter, to claim that composition is an identity relation. This thesis is known as Composition as Identity (CAI).

CAI can be meant more or less strictly, and the degree of strictness depends on which principles concerning identity are expected to also hold for composition. Identity is classically seen as the smallest reflexive relation on the universal domain, i.e., as the relation which everything bears to itself and to nothing else. It is uniquely identified by its Reflexivity and by the Indiscernibility of Identicals:

$$
\begin{aligned}
& \forall x(x=x) \\
& \forall x \forall y(x=y \rightarrow \forall P(P x \leftrightarrow P y))
\end{aligned}
$$

(Reflexivity of Identity) (Indiscernibility of Identicals)

Both these principles about identity are accepted by a wide majority of philosophers. For what concerns Reflexivity, it seems unclear how anything could fail to be identical to itself. As regards Indiscernibility (which — as we are going to see-is nonetheless slightly more controversial than Reflexivity), the hypothesis that identical entities have different properties seems dangerously similar to a contradiction.

To what extent (if any) is it possible to extend these principle about identity to the case of composition? Reflexivity is not especially problematic, inasmuch as in CEM everything indeed composes itself. The controversies mostly concern Indiscerniblity. The claim that in every case of composition the components and the composed entity share all their properties seems prima facie implausible. Donald Trump has some 
properties, such as that of being human, which the molecules composing him prima facie lack. Thus, it seems that in this case (as well as in many other similar cases) the components are not indiscernible from the composed entity. How can this appearance be made compatible with CAI's core claim that composition is an identity relation?

A goal of many defenders of CAI is to show that - in contrast with the above prima facie analysis of Trump's case-Indiscernibility can be extended to composition. For example, they claim that, once the distinction between distributive and collective predications is properly countenanced, the molecules are as collectively human as Trump individually is. Other strategies for extending Indiscernibility to composition are applied to trickier cases, such as those concerning cardinality: the appearance that Trump is one and not many, while the molecules are many and not one, would fade away once cardinalities are relativised to concepts or ways of counting.

In general, it seems that CAI, due to the pivotal role which Indiscernibility plays in it, ends up primarily concerning the features of components and composed entities. This seems to make the subject matter of CAI different from the subject matter of mereology; indeed, the axioms and theorems of CEM we have introduced above would not seem to concern the features of parts and wholes at all. Other metaphysical disciplines, such as the theories of supervenience and grounding, purport to characterise the links between features of the parts and of the wholes, quite independently of mereological issues.

In turn, and despite the popularity of Reflexivity of Identity and Indiscernibility of Identicals, identity is the subject matter of complex and long-standing philosophical controversies, which unavoidably interact with the mereological controversies about Extensionality and CAI. The classical characterisation we have presented above assimilates identity to logic, so that it is quite standard to consider first-order logic with identity as a unitary theory. As a consequence, also the theory of identity is expected to share with logic the distinctive marks of logicality, that is, the kind of neutrality and generality which, at least for those who are not radical logical pluralists, classical logic purportedly enjoys. In particular, identity is expected to be formal and topic neutral, that is, to work precisely in the same way no matter which kinds of entities it connects. Moreover, the Indiscernibility of Identicals, together with the Necessary Reflexivity of Identity, ${ }^{1}$ entails that every instance of identity holds necessarily.

While being a widely accepted standard, the resulting picture of identity has been and is contested by some alternative, non-standard doctrines, which state that identity is relative (Geach 1967; Deutsch and Garbacz 2018), indeterminate (Parsons 2000), modally contingent, temporally occasional (Gibbard 1975; Gallois 1998). The backers of these alternatives often argue that the Indiscernibility of Identicals should be restricted or rejected and/or that the assimilation of identity to logic should be resisted.

Now, on the one hand, suppose that we accept Extensionality and perhaps even CAI, thereby deeply entrenching identity in mereology. If we endorse the classical view of identity, are we thereby entitled to extend the features of identity (or at least some of them) to mereological relations? Is mereology a sort of logic (or is it a substantial metaphysical doctrine)? Is mereology as formal and topic neutral as logic and identity

\footnotetext{
1 Inasmuch as it is not clear how anything could fail to be identical with itself, Reflexivity of Identity plausibly also holds in its necessitated form: $\square \forall x(x=x)$.
} 
are? Do mereological relations always hold necessarily and permanently as classical identity does? In contrast, if non-standard theories of identity are adopted, how do the principles of CEM and CAI interact with them?

On the other hand, suppose that we incline towards non-classical mereologies, in which Extensionality is dropped, for instance, or that mereological principles are expected to be specific to categories of entities. Does this cut every tie between mereology and identity, or can non-classical mereologies be usefully compared with standard or non-standard philosophical theses about identity?

\section{Logicality, topic neutrality and analyticity}

According to the classical conception, identity is a paradigmatically formal, topic neutral and logical notion. We have seen in Sect. 2 that this classical conception is contested by many, but is nonetheless a sort of standard. Thus, if identity plays a pivotal role in mereology, then the mereological principles could be expected to inherit from identity the status of formality, or even of logicality, which the classical conception attributes to identity.

However, the concepts of formality, logicality and topic neutrality are in turn controversial, and these controversies reverberate on the status of mereological principles. The logical or non-logical, topic neutral or domain-specific status of mereological principles also has a potential impact on their epistemic status. For example, both Reflexivity of Identity and Indiscernibility of Identicals are hardly doubtful and might even be deemed implicit in the concept of identity. How can anything be thought as failing to be identical to itself or as failing to instantiate the features that it itself instantiates? Thus, these principles about identity could be analytic.

Then, if composition is an identity relation (as CAI claims), the principles about composition (such as Uniqueness of Composition and Unrestricted Composition) could also inherit a similar epistemic status, and thus be deemed analytic. The same might hold true for the principles about parthood. Indeed, some principles about parthood, such as Weak Supplementation, have often been considered analytic (see for example Simons 1987, p. 116).

The alleged analyticity of Weak Supplementation is discussed in Cotnoir's paper, Is Weak Supplementation Analytic? According to Cotnoir, many philosophers have thought of Weak Supplementation as analytic because they assumed a specific view of the relation of proper parthood. According to this view, a proper part of a certain whole (in contrast to an improper part) would be intuitively something less than the whole. Weak Supplementation would simply make the above intuition explicit, by claiming that, if $x$ is a proper part of $y$, then $y$ has something else in it (namely a part disjoint from $x$ ). This is the outstripping conception of proper parthood.

On the other hand, in recent years, some non-classical mereologies have been investigated, in which Weak Supplementation is not adopted (see for example Smith 2009). Despite their divergence from CEM, these theories seem to characterise in a certain way the same relation of parthood that CEM characterises in another way. This means that it is not plausible to think that a change of mereological theory leads to a change of subject, analogously to what happens (according to Quine) when a change 
of logic leads to a change of subject. If these mereological theories are genuinely about parthood, then there is no reason to think that Weak Supplementation (which these mereologies drop) is analytic with respect to parthood.

According to Cotnoir, these non-classical mereologies are crucially motivated by a different conception of proper parthood, namely the non-identity conception, according to which a proper part of a whole (in contrast to an improper part) is distinct from the whole (i.e. is non-identical to it). In CEM, as we have seen above, proper parthood is defined precisely in this way: $x P P y \equiv_{\operatorname{def}} x P y \wedge x \neq y$. Weak Supplementation also holds in CEM: this means that in CEM both the outstripping conception and the non-identity conception of proper parthood are adopted. However, in non-classical mereologies, the two conceptions can be separated, which is desirable in order to account for some kinds of dualism about the metaphysics of material constitution. Given a statue and the co-located portion of clay, neither of them outstrips the other. Nonetheless, according to dualists, the statue and the portion of clay are numerically distinct because they have different features; for example, only the portion of clay (not the statue) would continue to exist after the explosion of a powerful bomb, while only the statue (not the portion of clay) has aesthetic properties, such as being in Romanic style.

Which relation subsists between these two distinct entities? Given the non-identity conception of proper parthood, this relation might be that of proper parthood, but this is incompatible with the outstripping conception of proper parthood. Indeed, the statue does not outstrip the portion of clay. Thus, to account for dualism, it seems desirable to have a mereology that is faithful to the non-identity conception of proper parthood, but not to the outstripping conception. This drives the adoption of a non-classical mereology where Weak Supplementation (which is analytic only with respect to the outstripping conception, not to the non-identity conception) is dropped.

Guillon's Coincidence as Parthood makes even more explicit how some doubts about Weak Supplementation can emerge from specific metaphysical problems. According to Guillon, the rejection of Weak Supplementation is desirable because it paves the way for an interesting and scarcely explored solution to the so-called Amputation Paradox. Suppose that a person survives a radical surgical amputation, which removes everything except the brain. Before the amputation, the person was not identical to her brain. However, after the amputation, the person and the brain are spatially coincident, and would seem to have the same parts. Are they identical? This would make identity contingent, a solution that most philosophers are unwilling to swallow.

Guillon purports to develop a dualist approach to the person and to her brain, such that they are distinct both before and after the amputation. Both before and after the amputation, the brain would be a proper part of the person. After the amputation, the instance of proper parthood at stake violates Weak Supplementation: the person no longer has any part disjoint from the brain. According to Guillon, there is no strong reason to think that the brain ceases to be a proper part of the person just because the other parts are removed. The reasons why the person and the brain are distinct (i.e. having different properties and different histories before the amputation) continue to hold true. Cotnoir's non-identity conception of proper parthood is thus at work (even if Guillon does not employ this terminology). It is at work even when the person does 
not outstrip the brain any more. The non-identity conception of proper parthood leads Guillon to reject Weak Supplementation: after the amputation, the person has a single proper part, namely her brain.

Guillon tentatively endorses the following modalised version of Weak Supplementation: "If $x$ is a proper part of $y$, then possibly there is a $z$ disjoint from $x$ such that $x$ and $z$ are both proper parts of $y$ " (Sect. 5). In the above instance of the Amputation Paradox, it is indeed possible for the person to have another proper part besides the brain (this possibility was actualised before the amputation), and this possibility is a ground for distinguishing the person from the brain (indeed, the brain has no analogous possibility). Guillon's modalised version of Weak Supplementation is a new candidate for the role of analytic mereological principle, and certainly deserves further scrutiny.

In contrast, Maffezioli and Varzi's Intuitionistic Mereology discusses a kind of continuity between mereology and logic. Their paper concerns the problem of the impact of a change in the underlying logic on mereology. In particular, they show that the impact of replacing classical logic with intuitionistic logic concerns Extensionality, and - as a consequence - the identity conditions for complex entities. In general, the authors bring to light a certain degree of porosity between logic and mereology: if we weaken the underlying logic, we are forced to strengthen the proper axioms of mereology in order to regain an extensional theory. This kind of porosity might hint at the claim that mereology and logic share a similar status, from the perspective of logicality and perhaps also of analyticity. The same reasons that might lead us to doubt the analyticity of mereological principles (as in Cotnoir's paper) might lead us to doubt the analyticity of the underlying logical principles, given that the results that might be obtained by weakening mereological principles might also be gained by weakening the principles of the underlying logic. Some passing remarks about the continuity between mereology and logic can also be found in Bricker's Composition as Identity, Leibniz's Law, and Slice-Sensitive Emergent Properties (which we consider more extensively in Sect. 5). In continuity with a stance on the logicality of mereology which is often attributed to Lewis, Bricker explicitly writes: "I take mereology to be a part of logic, broadly construed" (Sect. 8). Bricker seems to incline towards the idea that mereology, on a par with logic, is a neutral tool for metaphysical disputes.

Azzano's paper, Structural Properties, Mereology and Modal Magic, expresses a radically different stance on the logicality and topic neutrality of mereology. Azzano opposes the idea that mereology is topic neutral as logic is often expected to be; indeed, he proposes that the adoption or the rejection of some mereological principles primarily depends on some specific features of the metaphysics of properties. According to Azzano, if structural properties (such as the property of being a methane molecule) are universals, then mereology should not be extensional; however, he concedes that this outcome might be avoided if structural properties are construed as tropes. As a consequence, the adoption of a principle (Extensionality), which is a theorem of CEM, is shown to depend on whether properties are at stake, and on whether these properties are universals or tropes. Thus, if Azzano is right, the category or the nature of the entities that are the relata of parthood and composition has a pivotal bearing on the choice of mereological principles.

Wallace's The Polysemy of 'Part' discusses some linguistic evidence that could be of interest for the opponents of the topic neutrality of mereology. Moreover, this evi- 
dence could support a form of mereological pluralism (the most influential defenses of mereological pluralism being Fine 2010; McDaniel 2014), in which different theories of parthood are needed for different categories of entities. Many introductions to mereology (e.g. Casati and Varzi 1999, ch. 2 and Lando 2017, ch. 1) begin by analysing excerpts of natural language about parthood. These introductions do not claim or presuppose that mereology should account for every true sentence about parthood in every human language. For example, it is usually conceded that a highly metaphorical love declaration such as "I am part of you, you are part of me, but we are different persons" should not be taken too seriously as a counterexample to the antisymmetry of parthood. The more modest expectation is that natural language is helpful in delimiting the subject matter of mereology, i.e in identifying the relations mereology is about.

Wallace's analysis of "part" and related terms in English suggests that if natural language is a reliable guide in delimiting the subject matter of mereology, then mereology is about several relations of parthood, thereby legitimising mereological pluralism. To show that "part" in English is a polysemous term, Wallace resorts to the so-called zeugma test: there is a zeugma when something odd or funny is perceived by a competent hearer in front of a single occurrence of a polysemous word. According to Wallace, the following parthood-related claim would be zeugmatically odd: "The soul is composed of three parts, and so is this puzzle". The zeugma would be the outcome of the polysemy of "parts".

Perhaps, the oddness of this specific claim might be explained simply by the fact that it is odd to speak about puzzles and souls in a single sentence. Suppose that someone says (without any part-related lexicon) "I enjoy solving puzzles, and my husband's soul is dirty". Her claim sounds odd; however, the oddness is not the upshot of any zeugma or polysemy, but of a lack of cohesion between the two subject matters of her claim. The source of the oddness of the sentence "I enjoy solving puzzles, and my husband's soul is dirty" might also be the source of the oddness of "the soul is composed of three parts, and so is this puzzle"; and no further explanation would then be needed. Nonetheless, in her paper, Wallace discusses several other cases in which "part" seems to be a polysemous term. In particular, these cases display a heterogeneity between the applications of "part" to concrete entities and its applications to abstract ones. These cases will likely further stimulate the investigation of the topic neutrality of mereology and the debate between mereological monism and mereological pluralism.

\section{Extensionality}

As we have anticipated in Sect. 2, Extensionality is a central, philosophically controversial principle of CEM. It is connected with the general, broadly nominalistic expectation that there is "no distinction of entities without distinction of content" (Goodman 1956, p. 202), and this expectation has been a primary motivation for the adoption of CEM as the true and exhaustive theory of parthood (see Lando 2017, ch. 5 for an analysis of the nominalistic motivations of mereology). However, this is simply mereological Extensionality. Other extensionality principles are discussed in other contexts. In particular, there are principles of extensionality for sets or plurali- 
ties, which claim that two sets or two pluralities are identical if and only if they have the same elements or members.

Some well-known connections exist among the various principles of extensionality. In general, these principles forbid distinctions among entities without a concomitant difference regarding what is in them, where the nature of this being in changes from case to case (being in consists in parthood for mereological wholes, in elementhood for sets and in being one of for pluralities). The extensionality for sets also performs a key role in Lewis's project of reducing set theory to mereology (Lewis 1991); if sets were allowed to be different despite having the same elements (in contrast to set-theoretic extensionality), then Lewis's idea about sets as mereological sums of the singletons of their elements would be a non-starter. In turn, extensionality for sets and extensionality for pluralities are also strictly connected to each other, inasmuch as two pluralities $x x$ and $y y$ are identical if and only if the sets $\{x x\}$ and $\{y y\}$ are identical as well.

In his paper, Extensionality for Fusions and Pluralities, Smid shows that mereological Extensionality and extensionality for pluralities also have an interesting, direct connection. The connection particularly concerns the arguments against these two principles of extensionality: the usual arguments against mereological Extensionality are structurally similar to $\mathrm{bad}$, unconvincing arguments against extensionality for pluralities. As a consequence, it is also possible to export to the mereological case the reasons why the bad arguments against extensionality for pluralities are indeed bad, thereby obtaining a defence of mereological Extensionality from the usual arguments.

Consider the well-known case of the Tinkertoy house; in contrast with the fusion of the Tinkertoys in it, the house would survive if one of the Tinkertoys were replaced. Dualists about constitution argue from this that the Tinkertoy house and the fusion of the Tinkertoys are discernible one from another and, owing to the Indiscernibility of Identicals, are also numerically distinct (Thomson 1983). Analogously, the Pink Floyd continued to exist even when Barrett had been replaced by Gilmour. However, Smid argues that this would be a poor reason to think that the members of Pink Floyd are not plurally identical to Barrett, Mason, Wright and Waters. The fact that at a certain point in time the denotation of the description "the members of Pink Floyd" changes does not show that there is any difference in the modal profile of what "the members of Pink Floyd" and "Barrett, Mason, Wright and Waters" denoted in 1969 (before the replacement of Barrett); they denoted a single plurality that always and necessarily is identical to itself. This legitimises us to react in the same way to the analogous Tinkertoy-like arguments against mereological Extensionality. In particular, we should be sceptical about arguments that proceed from linguistically based forms of discernibility to claims of numerical distinctness.

In a short passage in his paper (Sect. 7), Smid also tentatively points to an innovative way of dealing with anti-extensionalist intuitions about the Tinkertoy house and similar cases from the theory of material constitution, while retaining both mereological Extensionality and extensionality for pluralities. Dualists about constitution usually want to distinguish the structured house from the mere fusion or collection of the Tinkertoys, inasmuch as they would be discernible. However, the motivations for this distinction might be more straightforwardly served by the distinction between a single whole (the Tinkertoy house) on the one hand, and the plurality of Tinkertoys on the other. The Tinkertoy house abides by mereological Extensionality, while the 
plurality of Tinkertoys abides by extensionality for pluralities; what dualists deem to be the mere fusion or collection would actually be an extensional plurality of entities, while the Tinkertoy house would be the real, extensional mereological whole.

Extensionality plays a pivotal role in several other papers in the S.I., some of which have already been discussed here. For example, Azzano's Structural Properties, Mereology, and Modal Magic scrutinises the failure of Extensionality for universal structural properties, and Maffezioli and Varzi's Intuitionistic Mereology shows that Extensionality requires a stronger foundation, if the logical core of mereology is an intuitionistic one.

\section{Explanation}

The nominalistic motivations of mereological Extensionality can also be perceived as a request for a metaphysical explanation or ground for numerical distinctness; according to Extensionality, in the case of complex entities, the only admissible ground consists of their proper parts. No purely qualitative ground for numerical distinctness is admissible. An ideological anomaly of this approach relates to the fact that mereological atoms end up being treated in a special way. Since they have no proper part, their distinctness cannot be explained by their proper parts; thus, the explanation has to be different, and presumably qualitative. However, if qualitatively-grounded distinctness is admissible for mereological atoms, why should it be inadmissible for complex entities? Goodman's maxim "no distinction of entities without distinction of content" loses some of its appeal owing to its admitting exceptions.

However, numerical distinctness is strictly connected with its opposite-numerical identity. There are good reasons to doubt that the single instances of identity need to be explained at all. Each single instance of identity connects an entity with itself. Each of these instances might be explained by the fact that identity - as we have seen in Sect. 2-is in general reflexive, and this might in turn depend on the nature of identity itself; no explanation or ground specific to a certain self-identical entity is needed. If the single instances of identity need no explanation, then perhaps the single instances of distinctness (i.e., of non-identity) also do not need any explanation, and the problem of whether the explanation has to be exclusively mereological or not becomes moot (Fine (2016) and Carrara and De Florio (2018) are two recent works on grounding and identity criteria).

The general problem of whether single instances of relations need an explanation also emerges in mereology. Many classical theories about mereology have been formulated (e.g. by Goodman, van Inwagen and Lewis) when explanation, grounding and other hyperintensional relations were not yet at center stage; in contrast, these relations are now of primary concern for contemporary metaphysics (see Nolan 2014 for an overview).

Spencer deals with an instance of this general issue in his paper, On the Explanatory Demands of the Special Composition Question. He focuses on van Inwagen's Special Composition Question (SCQ) and on the expectation that a satisfying answer to it (i.e., a restriction of composition) should be explicative. Given an instance of composition in which some entities $t t$ compose an entity $u$, Spencer distinguishes between two prima 
facie plausible explananda for SCQ: a compositional fact (i.e., the fact that $t t$ compose $u$ ) and an ontological fact (i.e., the fact that $u$ - the composed entity-exists). SCQ might thus be given either a wide or a narrow explanatory scope. The explanatory scope is narrow if only the compositional fact is explained; the explanatory scope is wide if both the compositional fact and the ontological fact are explained. The distinction between an ontological fact and a compositional fact is typically hyperintensional, inasmuch as there is no possible scenario where one of them subsists and the other fails to subsist (indeed, $t t$ compose $u$ if and only if $u$ exists).

The answers to SCQ (including Unrestricted Composition) claim that, at certain more or less trivial conditions, there is something composed of certain entities. Thus, any instance of an answer to SCQ is an existentially quantified claim about the composed entity, and expresses an ontological fact, in Spencer's terminology. However, based on the recent literature on grounding, Spencer observes that an existential quantified claim is already grounded by the entity/entities in the domain of quantification that satisfies/satisfy the quantified formula. As a consequence, the additional groundwhich SCQ should provide if its explanatory scope is wide-would be redundant, and would risk generating an unacceptable form of overdetermination. For Spencer, this provides a reason to conclude that an answer to SCQ should be expected to ground only compositional facts and not ontological facts too.

There is also a more general sense in which explanation can be important for mereology. It is not always clear what mereology, as a philosophical discipline, is expected to explain: there are tendencies to push the explanatory duties of mereology well beyond the formal characterisation of parthood and cognate relations. Bricker's Composition as Identity, Leibniz's Law, and Slice-Sensitive Emergent Properties shows that these tendencies are rooted in CAI; indeed, as we have seen in Sect. 2, the claim that composition is an identity relation, via the Indiscernibility of Identicals, ends up greatly expanding the expected explanatory duties of mereology.

Let us see how. According to Bricker, the viability of CAI depends on whether it is possible to identify a principled restriction of the indiscernibility principle that holds for composition. Indeed, if composition did not respect a decently inclusive and wellmotivated indiscernibility principle, then it would not qualify as a genuine identity relation. Bricker explores the prospects of an indiscernibility principle restricted to properties ascribing a qualitative character. He shows that to circumscribe the domain of these properties it is pivotal to hold the view that, at the level of being, portions of reality are not characterised as being either singular or plural. The properties to which the indiscernibility principle is restricted should never depend on whether a certain portion of reality is described as either singular or plural.

Even when the neutrality of portions of reality with respect to the singular/plural distinction is conceded, the viability of CAI depends on whether there are slice-sensitive, qualitative emergent properties, that is, qualitative properties that are instantiated by a portion of reality sliced in a certain way and are not instantiated by that same portion of reality sliced in another way. For example, consider a world composed by some atoms, in a certain order. $a 1$ has a positive charge, $a 2$ has a negative charge, $a 3$ has a positive charge, $a 4$ has a negative charge, and so on. Now consider the net charge of this entire world; it possibly depends on how the atoms are sliced in pluralities. If they are sliced in couples such as $<a 1, a 2>,<a 3, a 4>\ldots$, then the 
net charge will depend on something like $(1-1)+(1-1)+\ldots$, and it will then be 0 . In contrast, if they are sliced in an initial single-membered plurality and then in couples $(<a 1>,<a 2, a 3>,<a 4, a 5>\ldots)$, then the net charge will depend on $1+(-1+1)+(-1+1)+\ldots$, and it will then be 1 . The net charge of such a world is a slice-sensitive emergent property, because it does not only depend on the properties of the parts, but also on how these parts are sliced in pluralities. If these kinds of properties exist, then the restriction to qualitative properties does not deliver an indiscernibility principle for composition, and the tenability of CAI is at risk. Thus, the tenability of CAI ends up depending on problems that go well beyond a narrow understanding of the explanatory duties of mereology and do not concern mereological relations at all.

\section{Modality}

Consider the following scenario. Yesterday at 9 p.m., the extremity $e$ of a man's left pinky toe nail was part of his body $b$. The fusion of $e$ and of the other parts of his body was $b$. At 9.01 p.m. he cut $e$ with nail clippers. As a consequence, $e$ stopped being part of $b$, and the fusion of $e$ with the remaining part of his body started being a scattered object, located partly on his couch and partly in his trash can. Parthood relations and composition relations seem to change in time, and to be also modally contingent; the man's shirt $s$ has a certain button $b_{1}$ as a part, but another button $b_{2}$ could have been used in the production process; it is seemingly contingent that $b_{1}$ is part of $s$ and compose $s$ together with the other parts of $s$, and it is equally contingent that $b_{2}$ is not part of $s$.

These claims about the occasionality and the contingency of parthood and composition depend on broad philosophical options in the philosophy of time and modality. If a kind of perdurantism about persistence is adopted, one might deny that there is inter-temporal identity, and therefore also deny that a single entity $(b)$ includes $e$ at a certain time and does not include $e$ at a later time. Thus, the option of denying the occasionality of mereological relations becomes viable. Analogously, if a theory of modal parts is adopted such that the shirt $s$ includes as parts all its counterparts in other possible worlds, as well as all the parts of these counterparts (and thus also a counterpart of the button $b_{2}$ ), then the option of denying the contingency of parthood and composition also begins looking less repugnant.

In any case, if identity plays a pivotal role in mereology, the problem of comparing the modal status of identity with the modal status of mereological relations emerges. Given Extensionality, the identity conditions for any complex entity exclusively depend on their proper parts. Thus, we may expect the instances of identity among complex entities to have the same modal and temporal status of the instances of proper parthood. Moreover, if composition is really an identity relation (as the backers of CAI claim), then composition and identity can be expected to be equally necessary (or equally contingent), and equally permanent (or equally occasional).

Barcan and Kripke's powerful argument, based on Necessary Reflexivity of Identity and on the Indiscernibility of Identicals, proves that any instance of identity holds necessarily. Every $x$ has indeed the property of being necessarily identical to itself, that 
is, to $x$. Every $y$ that is identical to $x$ has-owing to the Indiscernibility of Identicalsthis same feature. Thus, every $y$ that is identical to $x$ is necessarily identical to $x$. Analogously, the permanence of identity can also be proven.

Given the links between mereology and identity, there is an ensuing risk of making all the instances of parthood and composition equally necessary and permanent. In the extant literature this line of thought has been used as either a refutation of CAI (composition is contingent and identity is necessary; thus, composition is not an identity relation, contra CAI; Merricks 1999); or as a reason to integrate CAI with a specific theory of modality, such as the counterpart theory or the theory of modal parts (Bøhn 2014; Wallace 2014). However, the problem of the modal and temporal mismatch between identity and mereological relations is only seldom discussed in the literature about mereology and identity and surely deserves further scrutiny.

In our paper, Contingent Composition as Identity, we suggest a new variety of CAI that preserves both the contingency of composition and the necessity of standard identity, by flanking standard identity with a non-standard variety of identity, which is relative to possible worlds. In the variety of CAI that we outline, composition is an identity relation of the latter, world-relative variety. We show that the ensuing characterisation of the world-relative variety of identity is interestingly similar to some well-known doctrines of contingent and occasional identity. ${ }^{2}$

The modal and temporal mismatch between identity and mereological relations is also discussed or mentioned in several other papers. In his paper, No Universalism Without Gunk? Composition as Identity and the Universality of Identity, Lechthaler discusses the modal status of the Universality of Identity, which states that for every entity, something is identical to it. This principle is so basic and implicit in the tight connection between identity and quantification that it could not admit exceptions in any counterfactual scenario; thus, it is a necessary principle. Lechthaler shows that the Necessary Universality of Identity leads to hardly digestible consequences when it is applied to CAI, and to the peculiar one-many instances of identity that would connect any whole with its parts. The outcome would be that necessarily any entity is identical to many entities. Thus, necessarily everything has proper parts. The scenario in which everything has proper parts is usually called "gunk" (Lewis 1991, pp. 20-21). Reality would be necessarily gunky, while the existence of mereological atoms would be impossible. This outcome is especially troubling, because whether the world is atomic or gunky (a fact that is not settled by any theorem of CEM) is usually expected to be a contingent matter, to be assessed a posteriori and on the basis of scientific evidence.

Azzano's Structural Properties, Mereology, and Modal Logic (which we have already discussed in Sect. 3) also countenances (and finally rejects) the idea that if a structural property is the mereological fusion of some simpler properties and CAI is adopted, then we obtain an explanation of the necessary link between the instantiation of the structural property by a whole and the instantiation of the simpler properties by its parts. For example, we would thus explain why the fact that a certain whole is a water molecule is necessarily coordinated with the fact that one of its parts is an oxygen atom. The explanation would be that the property of being a molecule of

\footnotetext{
2 The similarity is especially tight with respect to the doctrine of occasional identity in Gallois (1998).
} 
water is composed of simpler properties, such as being an oxygen atom and being a hydrogen atom, and-modulo CAI and the Necessity of Identity-is also necessarily identical to these simpler properties. ${ }^{3}$

\section{Composition as identity: new problems}

There are many objections against CAI in the literature. Some of them have already been satisfactorily addressed by the defenders of CAI. For example, van Inwagen's syntactic objection to CAI (van Inwagen 1994), according to which a many-one identity claim about some entities to be fused and their fusion is syntactically ill-formed, is hardly relevant in the contemporary debate. As Cotnoir (2013, Sect. 1.2) has shown, even English has the resources to express many-one identities, through singularisation devices that build syntactically singular referential expressions out of semantically plural ones. These devices are the so-called free relatives, such as "what" in:

What the entities to be fused are identical to is their fusion.

The fusion of the entities to be fused is what they are identical to.

Moreover, some prima facie worrisome discernibility-based objections (i.e., objections that are based on the fact that a whole is discernible from its parts and thus cannot be identical to them, given the Indiscernibility of Identicals) can be deflated, once-as we have seen in Sect. 2 - the distinction between distributive and collective predications is considered. For example, the mere remark that a person's body is visible while the cells composing it are not visible is not a decisive objection against the identity of the body with the molecules: the molecules are not invisible distributively, but they collectively are as visible as the body is (see in particular Wallace 2011b).

In contrast, other problems for CAI (including some discernibility-based objections, as we shall show) are still far from being satisfactorily solved. Some papers in the S.I. deepen the analysis of these persisting problems, or introduce new objections to CAI.

Lipman's On Relativist Approaches to Many-One Identity focuses on the challenge of numerical discernibility, which states that the parts cannot be identical to the whole because the parts are many, while the whole is one. Some defenders of CAI (see in particular Bøhn (2014) and the paper by Bøhn in this S.I) ${ }^{4}$ address the problem of numerical discernibility by endorsing the broadly Fregean contention that cardinality attributions should be relativised to concepts. Thus, the Benelux, which is composed by Belgium, the Netherlands and Luxembourg, would be identical to them; both the Benelux on the one hand and Belgium, the Netherlands and Luxembourg on the other would be one relative to the concept of a multi-national entity and would be three relative to the concept of a country. These relativisation strategies are usually preferred to a primitivist strategy, according to which the compatibility of different cardinality ascriptions is a primitive, unexplained fact (see Spencer 2017).

Lipman shows that, at the end of the day, the relativisation strategies fail to explain how identical entities can be both one thing and many things. From this viewpoint,

\footnotetext{
3 This proposal, which Azzano rejects, has been defended in Hawley (2010).

4 See Carrara and Lando (2017) for a general analysis of the relativisation strategies.
} 
such strategies have no advantage over the primitivist alternative. Indeed, when two cardinalities are relativised to two concepts, the problem of why the portion of matter at stake can be conceptualised according to those two concepts, and not according to other concepts, arises. For example, the supporters of the Fregean relativisations explain the fact that a deck of cards is both 1 and 52 by relativizing these cardinalities to the concepts deck of cards and card respectively. However, this raises the problem of explaining why these two concepts are suitable for conceptualising that portion of matter, while the concept frog, for example, is unsuitable, as well as the problem of explaining why the concept card delivers precisely the cardinality 52, whereas the concept deck of cards delivers by contrast the cardinality 1. Lipman argues that this is no less puzzling than the primitivist naked claim that the same portion of matter is both 1 and 52 .

It might be asked whether the extant relativisation strategies really aspire to explain the compatibility of different cardinalities, and are therefore directly damaged by Lipman's analysis. Perhaps they simply assume that there are uncontroversial and independently motivated examples of single entities that are counted relatively to different concepts. For example, we commonly count the deck of cards in two ways, by specifying sortals or concepts (such as deck of cards and card); the two ways of counting deliver different cardinalities, 1 and 52. Once we admit that this happens in some cases, we have a precedent that legitimises us to dispose of the seeming numerical discernibility of any whole with respect to its parts. Lipman rightly states that the relativisers do not explain why certain concepts are suitable or unsuitable or why they deliver certain cardinalities. Arguably, this is not their duty qua defenders of CAI.

In his Is Composition Identity? Yi focuses on the difficult interaction between CAI and plural logic, that is, the logic concerning inferences among sentences that include plural quantified variables such as $x x$ and constants such as $t t$. As we have already seen when formalizing some mereological principles above, plural logic is very useful in mereology. The most worrying (for CAI) upshot of the interaction between CAI and plural logic is the so-called Collapse problem (Collapse henceforth; see Sider 2007; Yi 1999; Calosi 2018). Collapse consists of the following: CAI, together with some seemingly uncontroversial assumptions, would entail that, given some entities $t t$ that compose an entity $u$, all and only the entities that are parts of $u$ are one of $t t$. The right-to-left direction of Collapse (every entity that is one of $t t$ is part of $u$ ) follows from the definition of mereological composition (see (Composition - Definition) in Sect. 2). The left-to-right direction (every entity that is part of $u$ is one of $t t$ ) is the real cause of scandal, and can be proven as follows. Consider $v$, a part of $u$. The seemingly uncontroversial principle of Plural Covering states that, if an entity $x$ is part of an entity $y$, then there is a plurality of entities $z z$ such that $x$ is one of $z z$ and $y$ is the fusion of $z z$. Let $w w$ be such a plurality in the case of $v$ and $u$ : $v$ is one of $w w$ and $u$ is the fusion of $w w$. Given CAI, $u$ is identical both to both $t t$ and $w w$ (inasmuch as it is both the fusion of $t t$ and the fusion of $w w$ ). Thus, by transitivity of identity, $t t$ and $w w$ are also mutually identical. By the Indiscernibility of Identicals, whatever is one of $w w$ is also one of $t t: v$ is one of $w w$, and thus $v$ is also one of $t t$.

The mutual collapse of parthood and being one of is difficult to digest for most common-sense instances of mereological wholes, which are decomposable in multiple 
ways, and have, as a consequence, many more parts than the members of the single plurality of entities that they fuse. There are interesting attempts (see Sider (2014) and the paper by Loss in this S.I., which we discuss in Sect. 8 below) to block the reasoning by rejecting the principle of Plural Covering, which in turn (claiming that, given any instance of parthood, there is a certain plurality of parts) depends on the Comprehension Principle of the underlying plural logic. A typical formulation of the Comprehension Principle states that, if something satisfies a certain open formula, then there exists a plurality of entities that satisfy it. However, the Comprehension Principle lies at the core of plural logic, and it is methodologically controversial to curtail it in order to prevent CAI from entailing an undesirable consequence.

Arguably, plural logic serves many other purposes that are independent of CAI and in general of mereology. It can be used as an interpretation of second-order logic or as a tool to express principles of set-existence (see for example Linnebo 2018, chs. 1, 12). A weakened Comprehension Principle risks being inadequate for these non-mereological purposes of plural logic.

Furthermore, even if we prescind from other applications of plural logic and we only focus on mereology, plural logic might be expected to be a neutral tool by which $\mathrm{CAI}$ and, in general, mereological principles can be formulated and assessed, and this makes it inadvisable to change plural logic in order to avoid Collapse and defend CAI. Yi defends and develops this conservative methodological approach, in which plural logic is kept fixed and CAI is scrutinized on this basis. Yi shows that CAI leads to various problems, all connected with the above mentioned Collapse. As already shown by Calosi (2016), in particular, CAI leads to the claim that nothing has proper parts, so that the only cases of composition are those where the single, improper part of anything composes itself. CAI becomes the trivial, undisputed and uninteresting thesis that improper parthood is a genuine identity relation. As Yi underlines, no genuine advocate of CAI would be satisfied with such trivialisation; CAI is primarily intended by its advocates to hold for non-trivial cases of composition, in which some proper parts of something jointly compose it.

$\mathrm{Yi}$ also argues that if CAI is conjoined with Unrestricted Composition (the axiom of CEM that states that every plurality of entities has a fusion), then a form of monism follows: indeed, Mereological Universalism warrants that the mereological universe (i.e., an entity that is the fusion of everything) exists. However, in its above-mentioned trivialised form, CAI leads to the claim that nothing has proper parts; as a consequence, the universe also has only improper parts, that is, only parts that are identical to it. Thus, everything is identical to the universe, which ends up being the only existing entity (here is the ensuing monism).

This consequence may seem disastrous. However, it is important to remember that monism follows only if CAI and Mereological Universalism are combined with the perfectly standard form of plural logic adopted by $\mathrm{Yi}$; and that this combination will likely not be in the agenda of genuine backers of CAI, given that (as we have shown) many other, already well-known problematic consequences (e.g. Collapse itself) follow from the combination of CAI with standard plural logic.

Calosi's objections in his Is Parthood Identity? do not concern CAI directly, but the cognate thesis of Parthood as Identity, set forth by McDaniel (2014). In his paper McDaniel intended to explain several mereological principles (e.g. the Reflexivity of 
Parthood, the Transitivity of Parthood and Extensionality) directly in terms of the fact that parthood is an identity relation. Calosi shows that McDaniel's doctrine of Parthood as Identity encounters several difficulties, particularly regarding the interface between mereology and the theory of spatial location. McDaniel assumes a form of endurantism, where enduring entities instantiate properties in the spatio-temporal regions that they occupy. Relations are instantiated at the union of the space-time regions that the relata occupy. The relation of parthood - in particular - would be instantiated at the region occupied by the whole, inasmuch as the region occupied by the part is a sub-region of the region occupied by the whole, and as a consequence, the union of the two regions is simply the region occupied by the whole.

Identity is also instantiated relative to space-time regions. Indeed, McDaniel defines parthood in terms of this kind of relativised identity, as follows. $x$ is part of $y$ if and only if:

(a) $x$ is identical to $y$ at the region occupied by $y$;

(b) $x$ is not identical to $y$ at the smaller region occupied by $x$.

As it happens in other doctrines of relative identity, McDaniel's approach to identity is forced to limit the Indiscernibility of Identicals. In particular, McDaniel limits it to region-free properties (i.e., properties that do not encode any information about regions of space). Calosi shows that, in the light of the current literature about spatiotemporal location, McDaniel's occupation is better understood as exact location. The following problem ensues: suppose that $x$ is a part of $y$ at the region $r$, which is the region at which $y$ is exactly located. Given McDaniel's definition of parthood in terms of identity, this entails that $x$ is identical to $y$ at region $r$, where $y$ is exactly located. Given the restricted form of indiscernibility, $x$ also shares with $y$ at $r$ all the region-free properties. This means that $x$ instantiates all these properties at $r$. But this entails that $x$ is exactly located at $r$, so that the part and the whole end up being exactly located at the same region. This seems unacceptable, since any intuitively "smaller" proper part of a whole is not exactly located at the same spatio-temporal region at which the whole is located.

Moreover, Calosi suggests (and proves in the case of the Transitivity of Parthood) that, in McDaniel's proposal, the formal features of parthood are not really explained by the doctrine of Parthood as Identity, but by the formal features of the relation of spatial sub-region, where this relation is in turn modelled on set-theoretic inclusion. Given the well-known formal similarities between parthood and set-theoretic inclusion (those similarities that have led Lewis (1991) to hold the view that set-theoretic inclusion is indeed a case of parthood), this reduction is unsurprising. As a consequence, Parthood as Identity risks lacking its expected explanatory value.

\section{Composition as identity: new perspectives}

In the extant literature, CAI already comes in a variety of versions, which are sometimes distinguished according to their strength: for example, Weak CAI would merely claim that composition is analogous to identity, while stronger versions would claim that literally composition is identity (see Sider (2007), Wallace (2011a) and Cotnoir (2014) 
for a taxonomy of the versions of CAI). This variety notwithstanding, all the extant versions agree on the following claims:

(i) All the instances of composition, regardless of the size of the involved entities, are covered by CAI and are consequently assimilated to identity; CAI is thus expected to be absolutely general.

(ii) The underlying metaontology is Quinean, so that - in particular - the quantifiers employed in the formulation of CAI (every plurality of entities to be fused and every fusion are such that the fused entities are identical to their fusion) are ontologically committing.

(iii) CAI needs to address an issue of numerical discernibility (discussed above in Sect. 7) because the parts are numerically many and the whole is numerically one; in particular, the unity of the whole, which determines this issue, is a matter of cardinality.

We now show that three authors of the S.I.-namely Loss, Botti, Payton-have developed a new variety of CAI by abandoning (i), (ii) and (iii) respectively.

Loss's On Atomic Composition as Identity presents a variety of CAI in which (i) is dropped, inasmuch as CAI would only hold for the relation between any whole and its atomic parts. The main motivation for this restriction to atomic parts regards Collapse. According to Sider (2014), Collapse shows that there are "fewer pluralities than are normally expected" (p. 213). As we have shown in Sect. 7 in regard to Yi's paper, this approach is methodologically problematic, inasmuch as it ends up weakening plural logic in order to salvage CAI (a controversial thesis, rejected by most philosophers). Loss takes Sider's suggestion seriously, and advocates a version of the Comprehension Principle according to which there are only pluralities of mereological atoms, i.e., of entities without any proper part. Loss's weakening of the Comprehension Principle hits one of the philosophical roots of Collapse, and extirpates it. The root at stake relates to the fact that, if a whole can be divided into two pluralities of parts (in Loss's example, a house is both composed by a plurality of bricks and by a plurality of mereological atoms), then-by CAI and by the Transitivity of Identity-the two pluralities are mutually identical although their members are distinct (presumably, no mereological atom is a brick and no brick is a mereological atom). Given Loss's version of the Comprehension Principle, this undesirable outcome is avoided, because there are only pluralities of atoms.

Loss's restriction of CAI to the relation between a whole and its atomic parts is in consonance with one of the primary nominalistic motivations at the basis of CEM, namely Goodman's above quoted dictum "no distinction of entities without a distinction of content". For Goodman the content consisted precisely of the ultimate or atomic parts of entities. Given Loss's atomic variety of CAI, every complex entity is indeed identical to its atomic parts. As a result, the Goodmanian expectation that any difference between complex entities depends on their having different atomic parts finds a clear motivation.

As Loss acknowledges, something is odd in the mereological picture emerging from his paper; in his example the house is only the fusion of its atomic parts (and is one-many identical to these atomic parts). The bricks exist (each brick is identical to its atomic parts), and are parts of the house, but no plurality of bricks is such that the house 
is their fusion. One might want an explanation of this; if one considers all the bricks of which the house is made, one has in a sense covered the entire portion of reality than the house. How does it happen that nonetheless, one has at this point no plurality of bricks that compose the house? However, the feeling of oddness can perhaps be curbed by the fact that, departing from the bricks, one can arrive at something that plays the role of the plurality of bricks, albeit indirectly. In this way, it is also possible to account for the intuitive sense in which the bricks are the same portion of reality of the house. Indeed, consider the pluralities of atoms that are identical to each brick; and then unite all these pluralities, each of which is identical to a brick; the plurality that one obtains from this union is identical to the house. Thus, also in Loss's paper, the bricks are the same portion of reality of the house, albeit in this indirect way.

According to Botti in Composition as Analysis: the Meta-Ontological Origins (and Future) of Composition as Identity, a major problem of the extant varieties of CAI is that they rely on (ii). In particular, they adopt a Quinean metaontology in conjunction with CEM, and specifically with Unrestricted Composition. As a result, CAI is adopted on the background of a level ontological commitment to pluralities of entities and fusions: the purpose of CAI in this context, as it is especially clear in Lewis (1991, Sects. 3.4-3.6), is to ground the alleged ontological innocence of fusions in the context of CEM. According to Botti, this approach paves the way for the discernibility-based objections we have discussed above: if both the whole and the parts exist in the same way, then CAI is committed to the hardly defensible claim that a single thing is identical to many things, in spite of being numerically discernible from them.

In order to bring the debate forward, Botti proposes to lay aside CEM and the alleged ontological innocence of fusions, and also to change the underlying metaontology, in particular by admitting a form of expansionism inspired by Fine (2006) and Linnebo (2018). The domain of quantification is expandible, and its expansions also determine expansions of the domain of existents. To exist would not be tantamount to be real or fundamental: the domain of existents is relative to the stage of the expansion, while the reality or fundamentality of something depends on its absolute explanatory role. The initial stage of the expansion includes only the entire world, which would be the only fundamental entity: from there, the interpretationally possible expansions of the domain come to include various choices of its parts. These parts are the same portion of reality than the world, but provide additional metaphysical information about it. For this reason, the inclusion of parts in the domain of quantification would be a sort of analysis of the world. Thus, a better name for CAI would be Composition as Analysis.

Indeed, it is not only a matter of worse and better names: at the end of the day, Botti is willing to drop the claim that whole and parts are mutually identical, a claim which entails — via the Indiscernibility of Identicals - that they are indiscernible. Precisely because the expansions to parts bring new metaphysical information, the parts are discernible from the whole: in fact, there are predicates which express this information, and which are satisfied only by the parts, and not by the whole. Thus, Botti renounces to the identity and to the indiscernibility of wholes with their parts, and this explains the fact that her Composition as Analysis also avoids-as she convincingly arguesCollapse.

Botti contrasts metaphysical information with merely semantic information. Two referential expressions with different Fregean senses (such as "Hesperus" and "Phos- 
phorus") would carry different semantic information, but no different metaphysical information. In contrast, the expansions of the domain of quantification towards parts would carry a more substantial kind of information, i.e., metaphysical information. However, inasmuch as the parts are the same portion of reality than the whole, there are in both cases two different viewpoints on the same thing (where this thing is a star in the Fregean case and a portion of reality in Botti's case). These different viewpoints presumably also account for the semantic differences between the referential terms for the parts and the referential terms for the whole: thus, it is not clear whether the information which is added by the expansion is really non-semantic. More in general, the concept of metaphysical information surely needs further investigation, and Botti's application of this concept is likely to stimulate it.

In his How to Identify Wholes and Their Parts Payton ends up dropping iii), by revising the sense in which a whole is unitary. Through this revision, Payton sets forth a version of CAI called Composition Entails Identity (CEI). In CEI, the sense in which a certain whole is one is not numerical, but concerns the mutual cohesiveness of its parts. A whole (identical to its parts) would be one in the sense that it is unitary, in contrast to other pluralities of scattered parts, which lack this kind of unity. This substantial sense of unity is typical of the hylomorphist—broadly Aristotelian-tradition. As a result, Payton's defense of CAI is a rare and interesting attempt to make sense of CAI out of the context of CEM.

Payton also equates the oneness of wholes to the oneness of collective entities, such as teams and flocks: thus, a team would be one in the sense of being cohesive and interconnected, but would be many from the perspective of its cardinality. Payton is open to the idea that collective entities, such as teams, are also instances of manyone identity, so that the instances of composition would not be the only instances of many-one identity.

A problem with Payton's approach is that it does not clearly state how a whole should be counted. Payton seems to think that a whole is one only in the sense that it is unitary, and that, in contrast, a whole is not one in the more standard, cardinalityconnected sense that there is exactly an entity that is one of it. This means that, for what concerns its cardinality, a typical whole is many and not one; but how many is it? Consider the case of a chair, composed of four legs, a seat and a back: any of its parts is a whole, composed for example by some molecules. Thus, according to Payton, presumably, the seat of the chair is not one (in the cardinality-connected sense of oneness) either, but is as many as the molecules composing it. Thus, there is the risk that, in order to count the number of entities composing the chair, it is not enough to count the four legs, the seat and the back: we should go down until we reach a mereological bottom layer of atoms (at least if there is such a bottom layer). Only this bottom layer would reveal the true cardinality of the chair, which, far from being one in the cardinality-connected sense, it will be as many as the mereological atoms in it. These highly revisionist consequences about the cardinality of a chair (and of many other concrete objects) should be explicitly discussed. In general, it seems that Payton, while focusing on a non-cardinality related kind of oneness that any whole would enjoy, risks sidestepping (instead of solving) the problem of the cardinality of a whole. 
In contrast to Loss, Botti and Payton and as the title of his paper suggests, Bøhn in Composition as Identity: Pushing Forward does not present a new variety of CAI, but pushes forward the existing, radical version that he has already presented in some previous works (Bøhn 2009, 2014). According to Bøhn, composition abides by the same logical principles that govern standard identity. He also thinks that composition should be defined in terms of identity and that all the principles of CEM can be derived from an adequately strong variety of CAI. In the face of the objections to CAI stemming from the alleged indiscernibility of a whole from its parts, Bøhn advocates the idea that the properties that seem to make a whole discernible from its parts (such as cardinality, inasmuch as a whole is one and the parts are many) should be relativised to concepts and that, given this relativisation, the Indiscernibility of Identicals also holds for composition (this idea is also discussed in Lipman's paper that we have analyzed in Sect. 7). Bøhn suggests that the debate about CAI should move beyond these problems of indiscernibility, which have already been adequately answered by the advocates of CAI.

Bøhn's assessment of the debate about CAI and indiscernibility is arguably optimistic. There are many open problems with the extension of indiscernibility to composition, including the following three:

- it is far from clear which concepts are suitable for the role of property relativisers (see Koslicki (1997) about this problem);

- the relativization of cardinality ascriptions to concepts makes it difficult (or outright impossible) to paraphrase them in logical terms (Spencer 2017; Carrara and Lando 2017);

- some of the most promising strategies to extend the Indiscernibility of Identicals to composition risk relying on questionable presuppositions, such as atomism (Cotnoir 2013, fn. 13).

Concerning the first of the three above-cited problems (the identification of relativizing concepts), Bøhn’s paper indeed introduces a significant novelty, claiming that concepts should not be seen as mental entities or as components of propositions, but (in a Fregean vein) as functions from objects to truth values. These functions are allowed to exist in objective reality, and this legitimises their role in the defense of CAI. Consider the portion of reality corresponding to a chair: it is one with respect to the concept chair, and it is a very high number with respect to the concept molecule. For $\mathrm{B} \emptyset \mathrm{hn}$ both these concepts are objectively existing denizens of reality and cardinality can be ascribed to portions of reality only through the mediation of these functions. It is not completely clear how these functions work: it seems that, when the concept molecule is applied to the portion of reality, the output is not only the positive truth value (inasmuch as that portion of reality consists of molecules), but also a certain cardinality. How are these two outputs of the functions related one to another?

Bøhn's version of CAI seems to rely on a rather peculiar kind of ontology, in which concepts are no less important than objects. Presumably, concepts should not be identified with properties, their purpose being that of relativizing properties. Thus, entities fall under three basic categories: objects, properties and concepts. Portions of reality also seem to play a role, and are perhaps the fourth basic category of entities. 
Finally, Woods' Many, but One contributes to the cause of CAI by suggesting that CAI can be helpful in solving the so-called Problem of the Many (Unger 1980). In the instances of this problem, a myriad of entities seems to exist, where common sense would instead suggest that there is only one entity. In a variation of the standard example (which involves a cat on a mat), there are many different mereological fusions in the spatio-temporal region where we expect our umbrella to be: these mereological fusions include or exclude molecules of rain water or portions of tissue at the periphery of the umbrella. It seems arbitrary to pinpoint only one of those fusions as the only, true umbrella; but it also seems wrong to claim that a myriad umbrellas exist there.

Woods' solution consists of claiming that the many candidates for the role of the umbrella are collectively many-one identical to a single entity, and that this single entity is the best candidate to the role of the umbrella. Woods remarks that his approach is similar to a pre-extant solution to the problem of the many, namely Sider's maximality approach (Sider 2001). According to Sider, the best candidate for the role of the umbrella is the most inclusive among the candidates, i.e., the fusion that occupies the wider spatio-temporal region. This most inclusive candidate will also be the fusion of all the candidates, so that Woods and Sider agree in their identification of the best candidate. Nonetheless, Woods suggests that his own solution is superior to Sider's because it explains why the chosen candidate is the best. In Sider's maximality approach it is not clear why - so to say-bigger is better. In contrast, the fact of being such that all the candidates are collectively identical to it (this is the characterising feature of the best candidate in Woods' approach) would be a clear criterion for identifying the best candidate.

Acknowledgements A preliminary version of some of the papers in the S.I. has been presented at a conference we organised at the Scuola Normale Superiore in Pisa (Italy) on 3-5 July 2017. We thank all the participants to that conference. We also thank the reviewers, whose comments have been of great help in improving the content and the form of the papers. Finally, we are especially grateful to Catarina Dutilh Novaes both for her support, patience and helpful suggestions throughout this challenging process, and for the comments on this introduction.

\section{References}

Bøhn, E. (2009). Composition as identity: A study in ontology and philosophical logic. Amherst: PhD thesis, University of Massachusetts.

Bøhn, E. (2014). Unrestricted composition as identity. In A. Cotnoir \& B. Baxter (Eds.), Composition as identity (pp. 143-165). Oxford: Oxford University Press.

Calosi, C. (2016). Composition is identity and mereological monism. Philosophical Quarterly, 66(263), 219-235.

Calosi, C. (2018). Failure or boredom. The pendulum of composition as identity. American Philosophical Quarterly, 55(3), 281-291.

Carrara, M. \& De Florio, C. (2018). Identity criteria: An epistemic path to conceptual grounding. Synthese. https://doi.org/10.1007/s11229-018-1878-5.

Carrara, M., \& Lando, G. (2017). Composition and relative counting. Dialectica, 71(4), 489-529.

Casati, R., \& Varzi, A. (1999). Parts and places. Cambridge (MA): MIT Press.

Cotnoir, A. (2013). Composition as general identity. In K. Bennett \& D. Zimmermann (Eds.), Oxford studies in metaphysics (Vol. 8, pp. 295-322). Oxford: Oxford University Press.

Cotnoir, A. (2014). Composition as identity: Framing the debate. In A. Cotnoir \& D. Baxter (Eds.), Composition as identity (pp. 3-23). Oxford: Oxford University Press. 
Deutsch, H., \& Garbacz, P. (2018). Relative identity. In E. N. Zalta (Ed.), The Stanford encyclopedia of philosophy. Stanford: Metaphysics Research Lab, Stanford University (fall 2018 edition).

Fine, K. (2006). Relatively unrestricted quantification. In A. Rayo \& G. Uzquiano (Eds.), Absolute generality (pp. 20-44). Oxford: Oxford University Press.

Fine, K. (2010). Towards a theory of part. The Journal of Philosophy, 107(11), 559-589.

Fine, K. (2016). Identity criteria and ground. Philosophical Studies, 173(1), 1-19.

Gallois, A. (1998). Occasions of identity. Oxford: Clarendon Press.

Geach, P. (1967). Identity. The Review of Metaphysics, 21(1), 3-12.

Gibbard, A. (1975). Contingent identity. Journal of Philosophical Logic, 4, 187-221.

Goodman, N. (1956). A world of individuals. In I. M. Boschenski, A. Church, \& N. Goodman (Eds.), The problem of universals: A symposion (pp. 13-31). Notre Dame (Ind.): University of Notre Dame Press.

Hawley, K. (2010). Mereology, modality and magic. Australasian Journal of Philosophy, 88(1), 117-133.

Koslicki, K. (1997). Isolation and non-arbitrary division. Synthese, 112(3), 403-430.

Lando, G. (2017). Mereology. A philosophical introduction. London: Bloomsbury.

Lewis, D. (1991). Parts of classes. Oxford: Blackwell.

Linnebo, Ø. (2018). Thin objects. An abstractionist account. Oxford: Oxford University Press.

Llored, J.-P., \& Harré, R. (2014). Developing the mereology of chemistry. In C. Calosi \& P. Graziani (Eds.), Mereology and the sciences. Parts and wholes in the contemporary scientific context (pp. 189-212). Berlin: Springer.

McDaniel, K. (2014). Compositional pluralism and composition as identity. In A. Cotnoir \& D. Baxter (Eds.), Composition as identity (pp. 130-142). Oxford: Oxford University Press.

Merricks, T. (1999). Composition as identity, mereological essentialism, and counterpart theory. Australasian Journal of Philosophy, 77(2), 192-195.

Moltmann, F. (1997). Parts and wholes in semantics. New York: Oxford University Press.

Nolan, D. (2014). Hyperintensional metaphysics. Philosophical Studies, 171(1), 149-160.

Parsons, T. (2000). Indeterminate identity. Metaphysics and semantics. Oxford: Oxford University Press.

Sider, T. (2001). Maximality and intrinsic properties. Philosophy and Phenomenological Research, 63(2), $357-364$.

Sider, T. (2007). Parthood. The Philosophical Review, 116(1), 51-91.

Sider, T. (2014). Consequences of collapse. In A. Cotnoir \& D. Baxter (Eds.), Composition as identity (pp. 211-221). Oxford: Oxford University Press.

Simons, P. (1987). Parts. A study in ontology. Oxford: Clarendon Press.

Smith, D. (2009). Mereology without weak supplementation. Australasian Journal of Philosophy, 87(3), $505-511$.

Spencer, J. (2017). Counting on strong composition as identity to settle the special composition question. Erkenntnis, 82(4), 857-872.

Thomson, J. J. (1983). Parthood and identity across time. The Journal of Philosophy, 80(4), 201-220.

Unger, P. (1980). The problem of the many. Midwest Studies in Philosophy, 5(1), 411-467.

van Inwagen, P. (1994). Composition as identity. Philosophical Perspectives, 8, 207-220.

Wallace, M. (2011a). Composition as identity: Part 1. Philosophy Compass, 6(11), 804-816.

Wallace, M. (2011b). Composition as identity: Part 2. Philosophy Compass, 6(11), 817-827.

Wallace, M. (2014). Composition as identity, modal parts, and mereological essentialism. In A. Cotnoir \& D. Baxter (Eds.), Composition as identity (pp. 111-129). Oxford: Oxford University Press.

Yi, B.-U. (1999). Is mereology ontologically innocent? Philosophical Studies, 93(2), 141-160.

Publisher's Note Springer Nature remains neutral with regard to jurisdictional claims in published maps and institutional affiliations. 